International Journal of Modern Physics A

(C) World Scientific Publishing Company

\title{
QCD COLLISIONAL ENERGY LOSS IN AN INCREASINGLY INTERACTING QUARK GLUON PLASMA
}

\author{
M. B. GAY DUCATI \\ Instituto de Física, Universidade Federal do Rio Grande do Sul \\ Caixa Postal 15051, CEP 91501-970, Porto Alegre, RS, Brazil \\ beatriz.gay@ufrgs.br \\ V. P. GONÇALVES \\ Instituto de Física e Matemática, Universidade Federal de Pelotas \\ Caixa Postal 354, CEP 96010-090, Pelotas, RS, Brazil \\ barros@ufpel.edu.br \\ L. F. MACKEDANZ $a, b$ \\ a Centro de Ciências Exatas e Tecnológicas, Universidade Federal de Pelotas \\ Campus Caçapava do Sul \\ Rua Rui Vieira Machado s/n ${ }^{\circ}$, CEP 96570-000, Caçapava do Sul, RS, Brazil \\ ${ }^{b}$ Instituto de Fúsica, Universidade Federal do Rio Grande do Sul \\ Caixa Postal 15051, CEP 91501-970, Porto Alegre, RS, Brazil \\ luiz.mackedanz@ufrgs.br
}

\begin{abstract}
The discovery of the jet quenching in central $\mathrm{Au}+\mathrm{Au}$ collisions at the Relativistic Heavyion Collider (RHIC) at Brookhaven National Laboratory has provided clear evidence for the formation of strongly interacting dense matter. It has been predicted to occur due to the energy loss of high energy partons that propagate through the quark gluon plasma. In this paper we investigate the dependence of the parton energy loss due to elastic scatterings in a parton plasma on the value of the strong coupling and its running with the evolution of the system. We analyze different prescriptions for the QCD coupling and calculate the energy and length dependence of the fractional energy loss. Moreover, the partonic quenching factor for light and heavy quarks is estimated. We found that the predicted enhancement of the heavy to light hadrons $(D / \pi)$ ratio is strongly dependent on the running of the QCD coupling constant.
\end{abstract}

Keywords: Quark gluon plasma; Collisional energy loss; Running coupling constant

PACS numbers: 12.38.Mh; 13.85.N; 25.75.-q

\section{Introduction}

Relativistic heavy ion collisions provide an opportunity to study the QCD properties at energy densities about thirty times higher than the density of the atomic nuclei 1213. In these extreme conditions a deconfined state of quarks and gluons, the Quark Gluon Plasma (QGP), is expected to be formed in the early stage of the collision. Recently, the discovery of jet quenching in central $\mathrm{Au}+\mathrm{Au}$ collisions 
at the Relativistic Heavy-ion Collider (RHIC) at Brookhaven National Laboratory has provided clear evidence for the formation of strongly interacting dense matter. Detailed analysis indicate that the suppression of the single hadron spectra at high $p_{T}$, the disappearance of back-to-back correlation of high $p_{T}$ hadrons and the azimuthal anisotropy of high $p_{T}$ hadron spectra in noncentral collisions are caused by parton energy loss (For a recent review see, e.g, Ref. 4). Basically, the high parton density produced in heavy ion collisions could induce a large amount of energy loss while hard partons produced in the initial stage of the collision propagate through the fireball, due to the interactions of the hard partons with the medium.

The total energy loss of a particle in a medium can be decomposed into a collisional and a radiative contribution. While the first one originates from the energy transfer to the medium particles, the latter one is caused by radiation from the fast particle. At large energies one expects that radiative energy loss becomes much larger than the collisional one, as in the electromagnetic case. However, at lower energies these two processes can contribute equally, with the collisional one being the dominant for small values of the parton energy. Currently, an open question is to quantify the contribution of each process in the RHIC kinematical region. In particular, in the last few years the understanding of parton energy loss by gluon bremsstrahlung has been extensively developed (For recent reviews see, e.g., Refs. $5|6| 7 \mid 8$ ). Recent works have analyzed the energy $9 \mid 10$, color charge and mass dependence of radiative parton energy loss 11/12|13, as well as its non-Abelian feature 14. A basic characteristic from these works is the assumption that the radiative energy loss dominates disregarding the collisional one. However, recent studies in charm quark thermalization 15/16, quenching of hadron spectra $17 \mid 18$ and the elastic parton energy loss including all $2 \rightarrow 2$ processes 19 indicate that in the RHIC kinematical region it is far from clear that radiative energy loss dominates over collisional energy loss.

Another important aspect is that in general the approaches for radiative and collisional energy loss start from the assumption that the properties of the medium and its interactions with the energetic parton projectile do not change with time (For a discussion of the medium evolution see, e.g. Refs. 20|21). However, in nucleus nucleus collisions at collider energies, the produced hard partons propagate through a rapidly expanding medium. The density of scattering centers is expected to reach a maximum value at the plasma formation time, $\tau_{0}$, and then decrease with time $\tau$ rapidly due to the strong longitudinal expansion. We begin following the progress of the quark at $\tau=\tau_{0}$, where it begins a process of scattering and diffusion in the plasma which causes a loss of the initial momentum and relaxation towards the thermal velocity. The scenario which we assume in this paper for the equilibration has been proposed some years ago 22 (See also Ref. 23) and consider that the interactions in the plasma of quarks and gluons get stronger with the time because the average parton energy drops due to the expansion of the system. The basic idea comes from the feature that the QGP is not a static medium, but it is cooling 
while partons propagate through. Consequently, the scale of the coupling changes with the evolution of the system, and this feature motivates the calculation of the observables assuming the running coupling. In this paper we will assume that the temperature is the dominant scale and consequently will control the running of the QCD coupling. The standard procedure is to take the solution for the running coupling from the renormalization group equation, which to lowest (1 - loop) order is given by:

$$
\alpha_{s}(\bar{\mu})=\frac{12 \pi}{\left(33-2 n_{f}\right) \ln \frac{\bar{\mu}^{2}}{\Lambda_{\overline{M S}}^{2}}},
$$

and put the renormalization point $\bar{\mu}$ proportional to the first Matsubara frequency, $\bar{\mu} \propto 2 \pi T$, which for massless quarks is the only dimensionfull quantity inherent to the theory. Moreover, the strong coupling $\alpha_{s}$ also depends on the scale parameter of the modified minimal subtraction scheme $\Lambda_{Q C D}$. However, it is not evident that temperature is the relevant scale at the energies currently accessible in heavy ion physics, where the temperatures reached are only moderately larger than the phase transition temperature. As the temperature drops over the lifetime of the QGP, $\alpha_{s}$ should also vary during the equilibration and the evolution of the plasma. In order to simplify our considerations we model the space-time evolution of the quark-gluon plasma by the Bjorken scenario with boost invariant longitudinal expansion and conserved entropy per rapidity unit 24 . This implies that $T=T_{0}\left(\frac{\tau_{0}}{\tau}\right)^{\frac{1}{3}}$, where $T_{0}$ is the initial temperature at the initial time $\tau_{0}$. We neglect the transverse expansion of the system. Furthermore, we will consider in what follows two prescriptions for the temperature dependence of the running coupling constant. First we will assume

$$
\alpha_{s}(T)=\frac{6 \pi}{\left(33-2 n_{f}\right) \ln \left[\left(19 T_{c} / \Lambda_{\overline{M S}}\right)\left(T / T_{c}\right)\right]},
$$

where $T_{c} / \Lambda_{\overline{M S}}=1.78 \pm 0.03$. This prescription, which we denote by thermal $\left[\alpha_{s}^{(t h)}\right]$, has been used in, e.g., Refs. 25|26. Furthermore, we also consider that $\alpha_{s}$ can be given by

$$
\alpha_{s}(T)=\frac{2.095}{\frac{11}{2 \pi} \ln \left(\frac{Q}{\Lambda_{\overline{M S}}}\right)+\frac{51}{22 \pi} \ln \left[2 \ln \left(\frac{Q}{\Lambda_{\overline{M S}}}\right)\right]},
$$

with $Q=2 \pi T$. This parametrization of the strong coupling has been obtained from recent results in the lattice 27 . We denote this prescription by $\alpha_{s}^{(\text {lat })}$.

In Fig. 1 we present the temperature dependence of $\alpha_{s}$ predicted by these two prescriptions. We use $T_{0}=375 \mathrm{MeV}$ and $t_{0}=0.33 \mathrm{fm}$ which are reliable values for RHIC energies 15 . The horizontal lines characterize the constant values of the QCD coupling: (a) $\alpha_{s}=0.3$, as in previous calculations 18 , (b) $\alpha_{s}=0.2$, which is a typical value for the coupling constant and (c) $\alpha_{s}=0.5$ for comparison. We can see that there is a large difference in normalization and shape between the two prescriptions. Comparing the results, the lattice motivated prescription given in Eq. (3) $\left[\alpha_{s}^{(l a t)}\right.$ in the figure] provides the largest $\alpha_{s}$ value at small temperatures. The fixed values 
$\alpha_{s}=0.2$ and 0.3 lie between these two prescriptions. On the other hand, a fixed value of $\alpha_{s}=0.5$ is larger than the lattice prescription at large temperatures. It is important to emphasize that if the prescriptions proposed in Refs. $22 \mid 28$ are used we obtain a behavior for the running coupling similar to $\alpha_{s}^{\text {(lat) }}$.

As the temporal development of the coupling constant modifies the behavior of several QGP signatures 22|23|28|29|30, we can expect a similar effect in the estimates of the energy loss of a parton propagating in a QGP. Our main goal in this paper is to estimate the influence of the running coupling constant in the collisional energy loss, presenting a reanalyzes of the studies from Refs. $17 / 18 \mid 31 / 32$, and an estimate of the quenching factor $Q\left(p_{\perp}\right)$ for light and heavy quarks (See discussion in Sect. 2). We postpone for a future publication the study of this effect in the radiative energy loss (For a short discussion see Ref. 33 ).

A comment is in order here. In our studies we consider that the fractional energy loss is given in terms of the Bjorken formula 34 as generalized in Refs. 35|36. Recently, Peshier 37 has advocated that the collisional energy loss is an observable for which loop corrections to the tree level approximation are essential. In particular, if the running of the coupling constant is taken into account the collisional energy loss becomes independent of the jet energy in the high energy limit $(E \gg T)$. It implies a distinct temperature dependence for the elastic energy loss when compared to the Bjorken formula and a larger value for the mean energy loss per length. If the formalism considered in this paper is reanalyzed assuming as input the Peshier's prediction, we can expect a larger contribution of the elastic energy loss. Consequently, the results presented here can be considered as a lower bound for the contribution of the elastic energy loss for the parton quenching.

This paper is organized as follow. In next section we define the partonic quench-

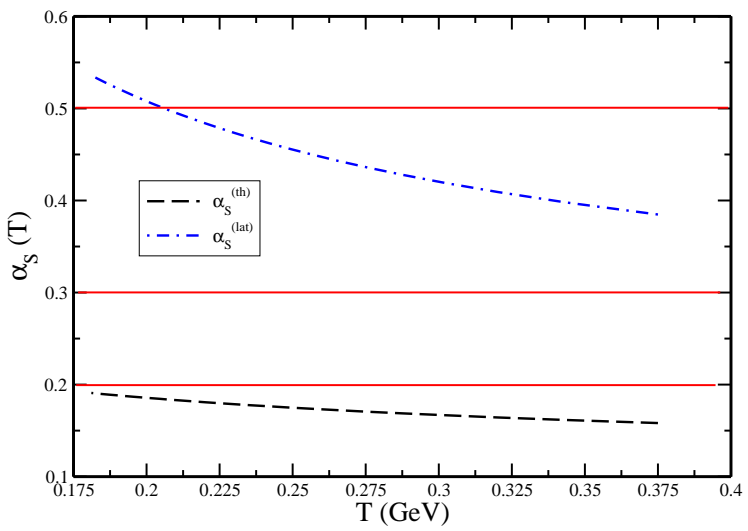

Fig. 1. Temperature dependence of the QCD coupling considering two different prescriptions. See text. 
ing factor $Q\left(p_{\perp}\right)$ and present its relation with the energy loss $\Delta E$. Furthermore, the Fokker-Planck equation is derived and its solution is obtained. In Sect. 3 we present our predictions for the time evolution of the drag coefficient for the propagation of light and heavy quarks considering different prescriptions for the QCD coupling, as well as our results for the energy and length dependence of the fractional energy loss. Moreover, our predictions for the quenching factor for light and heavy quarks are also presented. Finally, in Sect. 4 we present some remarks and our main conclusions.

\section{Parton Propagation in a QGP and the Partonic Quenching Factor}

Lets start our discussion considering the hadron production in nucleus-nucleus collisions. Due to the interactions of the produced parton with the medium, it loss an additional energy fraction $\Delta E$ while escaping the collision. Consequently, the inclusive transverse momentum spectra of the particles produced in nucleus - nucleus collisions will be modified with respect to hadron - hadron collisions. In general, the hadron formation is described in terms of parton recombination and/or by the fragmentation of the energetic partons. In particular, it is expected that for $p_{\perp}>5$ $\mathrm{GeV}$ the hadrons are dominantly produced by fragmentation. In what follows we assume that fragmentation is the dominant process of hadron formation and that it occurs after the parton has left the comoving medium. It allows to analyze the energy loss effects directly in the $p_{\perp}$ spectrum of the scattered partons. In Refs. $38 \mid 39$ the effect of the radiative parton energy loss in the $p_{\perp}$ distribution has been estimated. Here we extend this approach for collisional energy loss. Following Refs. $38 \mid 39$ we assume that the $p_{\perp}$ spectrum is given by:

$$
\frac{d N^{\mathrm{med}}}{d^{2} p_{\perp}}=\int d \epsilon D(\epsilon) \frac{d N^{\mathrm{vac}}\left(p_{\perp}+\epsilon\right)}{d^{2} p_{\perp}} \equiv Q\left(p_{\perp}\right) \frac{d N^{\mathrm{vac}}\left(p_{\perp}\right)}{d^{2} p_{\perp}}
$$

where $\frac{d N^{\mathrm{vac}}\left(p_{\perp}+\epsilon\right)}{d^{2} p_{\perp}}$ is the transverse momentum distribution in elementary partonparton collisions, evaluated at a shifted value $p_{\perp}+\epsilon$, and $D(\epsilon)$ is the probability distribution in the energy $\epsilon$ lost by the partons in the medium through collisions. Moreover, $Q\left(p_{\perp}\right)$ is the medium dependent quenching factor. In Ref. 38 the authors have demonstrated that in a realistic calculation of the quenching, the knowledge of the full probability distribution is actually required. However, as our goal is to study the effect of the running of coupling constant in the estimates of the quenching factor, we will assume, following Ref. 39 , that the quenching can be modeled in terms of the mean energy loss. Therefore, in this work we will calculate the partonic quenching factor assuming that it can be approximated by 39

$$
Q\left(p_{\perp}\right)=\frac{d N^{\mathrm{med}}}{d^{2} p_{\perp}} / \frac{d N^{\mathrm{vac}}}{d^{2} p_{\perp}}
$$


where

$$
\frac{d N^{\mathrm{med}}}{d^{2} p_{\perp}}=\frac{1}{2 \pi^{2} R^{2}} \int_{0}^{2 \pi} d \phi \int_{0}^{R} d^{2} r \frac{d N^{\mathrm{vac}}\left(p_{\perp}+\Delta E\right)}{d^{2} p_{\perp}},
$$

$R$ is the nuclear radius, $\phi$ is the angle between the velocity and the radius vector of the parton [See Eq. (18)] and $\Delta E$ is the total energy loss by partons in the medium.

In order to calculate $\Delta E$ we will use the approach proposed by Svetitsky in Ref. 31, which considers the Brownian motion of a parton in a thermal bath, governed by the Fokker-Planck equation. This approach has been used to estimate the diffusion of charm quarks in a quark - gluon plasma 31132 (See also Ref. 43 ) and its equilibration 44/45, as well as to calculate the quenching of light 17 and heavy quarks 15]16[18. In this approach one starts from the Boltzmann equation for the distribution function $f(x, p)$ and assume that there is no external force acting on the quark and that the phase space distribution $f$ does not depend on the position of the quark. Furthermore, assuming the scattering process to be dominated by small momentum transfers, one arrives at a Fokker-Planck equation describing the evolution of $f$ in the momentum space. We then investigate the time evolution of the Fokker-Planck equation in a thermally evolving QGP. The main simplifying assumption in the approach is the momentum-independence of the drag and diffusion coefficients. In what follows we present the main formulae of this approach. However, we do not repeat here the details of the calculations and refer the reader to the original works $31 / 3217 / 18$. 46

The Boltzmann equation in the relativistically covariant form can be written as

$$
p^{\mu} \partial_{\mu} f(x, p)=C\{f\},
$$

where $p^{\mu}=(E \boldsymbol{p}, \boldsymbol{p})$ is the four-momentum of the test quark and $f$ is its phase-space density and $C\{f\}$ is the collision term. Following Refs. $31|32| 17 \mid 18$ we assume that: (a) the hydrodynamical evolution can be described by the Bjorken scenario, which implies that it is valid to assume that the plasma is uniform and consequently the phase space density of the quark is independent of $\vec{x}$, and (b) the collision term is given by the elastic collisions of the test quark with other quarks, antiquarks and gluons in the system. Using the Landau approximation and restringing the analyzes to the one-dimensional problem one obtain $31 / 32|17| 18$

$$
\frac{\partial f}{\partial t}=\frac{\partial}{\partial p}\left[\mathcal{T}_{1}(p) f\right]+\frac{\partial^{2}}{\partial p^{2}}\left[\mathcal{T}_{2}(p) f\right]
$$

which is the Landau kinetic equation, with the transport coefficients given by

$$
\begin{aligned}
& \mathcal{T}_{1}(p)=\int d k w(p, k) k=\frac{\langle\delta p\rangle}{\delta t}=\langle F\rangle, \\
& \mathcal{T}_{2}(p)=\frac{1}{2} \int d^{3} k w(p, k) k^{2}=\frac{\left\langle(\delta p)^{2}\right\rangle}{\delta t},
\end{aligned}
$$

where $w(p, k)$ sums the rate of collisions of a test particle with the partons of the medium and $\langle F\rangle$ is the average force acting on the test particle. If we consider 
that the background heat bath is constituted of a large amount of weakly coupled particles in thermal equilibrium at a temperature $\mathrm{T}$, with some non-thermal but homogeneously distributed particles due to the fluctuations, the problem can be simplified 46 assuming that the equilibrium of the bath will not be disturbed by the presence of these few non-thermal particles. Due to its small number, one can also assume that they will not interact among themselves, only with particles of the thermal bath. Consequently, one can replace the distribution functions of the collision partners of the test particle by their Fermi-Dirac or Bose-Einstein distributions and Eq. (8) reduces to the Fokker-Planck equation.

The transport coefficients $\mathcal{T}_{1}$ and $\mathcal{T}_{2}$ can be expressed in terms of the drag coefficient of quark $\mathcal{A}$, which is almost independent of momentum $p$ as shown in Refs. $31 \mid 32$. It allows to write the Fokker-Planck equation as follows $31|32| 17 \mid 18$

$$
\frac{\partial f}{\partial t}=\mathcal{A} \frac{\partial}{\partial p}(p f)+\mathcal{D}_{F} \frac{\partial^{2} f}{\partial p^{2}}
$$

where $\mathcal{D}_{F}$ is the diffusion coefficient, which is given by $\mathcal{D}_{F}=\mathcal{A} T^{2}$ if we assume that the momentum $p$ can be approximated by the temperature $T$ of the system and the coupling between the Brownian particle and the bath is weak 46 . The Eq. (10) describes the evolution of the momentum distribution of a test particle undergoing Brownian motion. The solution from Eq. (10) has been obtained in Refs. 17/18, assuming as boundary condition $f(p, t) \stackrel{t \rightarrow t_{0}}{\longrightarrow} \delta\left(p-p_{0}\right)$ and using the method of characteristics 17 . It is given by 1718

$$
f(p, L)=\frac{1}{\sqrt{\pi \mathcal{W}(L)}} \exp \left[-\frac{\left(p-p_{0} e^{-\int_{0}^{L} \mathcal{A}\left(t^{\prime}\right) d t^{\prime}}\right)^{2}}{\mathcal{W}(L)}\right],
$$

where $\mathcal{W}(L)$ is given by

$$
\mathcal{W}(L)=\left(4 \int_{0}^{L} \mathcal{D}_{F}\left(t^{\prime}\right) \exp \left[2 \int_{0}^{t^{\prime}} \mathcal{A}\left(t^{\prime \prime}\right) d t^{\prime \prime}\right] d t^{\prime}\right)\left[\exp \left(-2 \int_{0}^{L} \mathcal{A}\left(t^{\prime}\right) d t^{\prime}\right)\right],
$$

and is the probability distribution in momentum space. In the previous equation the length of the expanding plasma is assumed as being the maximum time limit.

From Eq. (11) we can estimate the mean energy of the parton due to elastic collisions after traversing a distance $L$. It is given by

$$
\langle E\rangle=\int_{0}^{\infty} E f(p, L) d p .
$$

Then the average energy loss due to elastic collisions in the medium will be given by

$$
\Delta E=E_{0}-\langle E\rangle,
$$

where $E=m_{\perp}=\sqrt{p_{\perp}^{2}+M^{2}}$ at the central rapidity region, $y=0$. Consequently, in order to estimate the average energy loss it is necessary to calculate Eq. (11) in 
terms of the drag coefficient of a quark. Following Refs. 31/32, we approximate the drag coefficient by its average value,

$$
\langle\mathcal{A}(p, t)\rangle=\mathcal{A}(t)=\left\langle-\frac{1}{p} \frac{d E}{d L}\right\rangle,
$$

which is directly dependent of the energy loss rate $d E / d L$. The above approximation is reasonable up to moderate momentum values $(p \leq 15 \mathrm{GeV}) \underline{18}$.

The energy loss rate in the QGP due to elastic collisions with high-momentum transfer have been originally estimated by Bjorken 34 and recalculated in Refs. 40|35/36/41 taking into account the loss with low-momentum transfer dominated by the interactions with plasma collective modes in the hard thermal loop approximation 42 . In particular, in Ref. 36 the authors have estimated the energy loss for heavy quarks and in Ref. $\frac{35}{}$ for light partons. For heavy quarks and in the domain $E<<M^{2} / T$, it reads

$$
-\frac{d E}{d L}=\frac{8 \pi \alpha_{s}^{2} T^{2}}{3}\left(1+\frac{n_{f}}{6}\right)\left[\frac{1}{v}-\frac{1-v^{2}}{2 v^{2}} \ln \left(\frac{1+v}{1-v}\right)\right] \ln \left[2^{\frac{n_{f}}{6+n_{f}}} B(v) \frac{E T}{m_{g} M}\right]
$$

whereas for $E>>M^{2} / T$, it is

$$
-\frac{d E}{d L}=\frac{8 \pi \alpha_{s}^{2} T^{2}}{3}\left(1+\frac{n_{f}}{6}\right) \ln \left[2^{\frac{n_{f}}{2\left(6+n_{f}\right)}} 0.92 \frac{\sqrt{E T}}{m_{g}}\right]
$$

where $n_{f}$ is the number of quark flavors, $\alpha_{s}$ is the strong coupling constant, $m_{g}=$ $\sqrt{\left(1+n_{f} / 6\right) g^{2} T^{2} / 3}$ is the thermal gluon mass, $E$ is the energy and $M$ is the mass of the quark. $B(v)$ is a smooth velocity function, which can be taken approximately as $0.7 \underline{36}$. For light quarks we use the expression (17) and set $M=0$ in the calculations. Inclusion of the diagrams other than the $t$ channel increase the energy loss rate for light quarks by a factor 219 .

\section{Results and Discussion}

At the energies (temperatures) which we are interested in this paper the drag coefficient $\mathcal{A}$ for partons propagating in a plasma can be calculated using Eq. (15) and the expressions for elastic energy loss given by Eqs. (16) and (17). The average over the momentum is made using the Boltzmann distribution. As we assume the Bjorken scenario for the hydrodynamics evolution 24 , the time dependence of the temperature is given by $T(t)=t_{0}^{1 / 3} T_{0} / t^{1 / 3}$, where $t_{0}$ and $T_{0}$ are, respectively, the initial time and temperature at which the background of the partonic system has attained local kinetic equilibrium. The time dependence from the drag coefficient is directly associated with this evolution for the temperature, which decreases with time as the system expands. We assume as maximum time limit for the evolution the length of the plasma $L$. These approximations has been considered in Refs. $17 / 18$ which we would like compare our results. Moreover, we assume $T_{0}=375 \mathrm{MeV}$ and $t_{0}=0.33$ $\mathrm{fm}$ for RHIC energies as in Ref. 15 . The results for the time dependence of the drag 

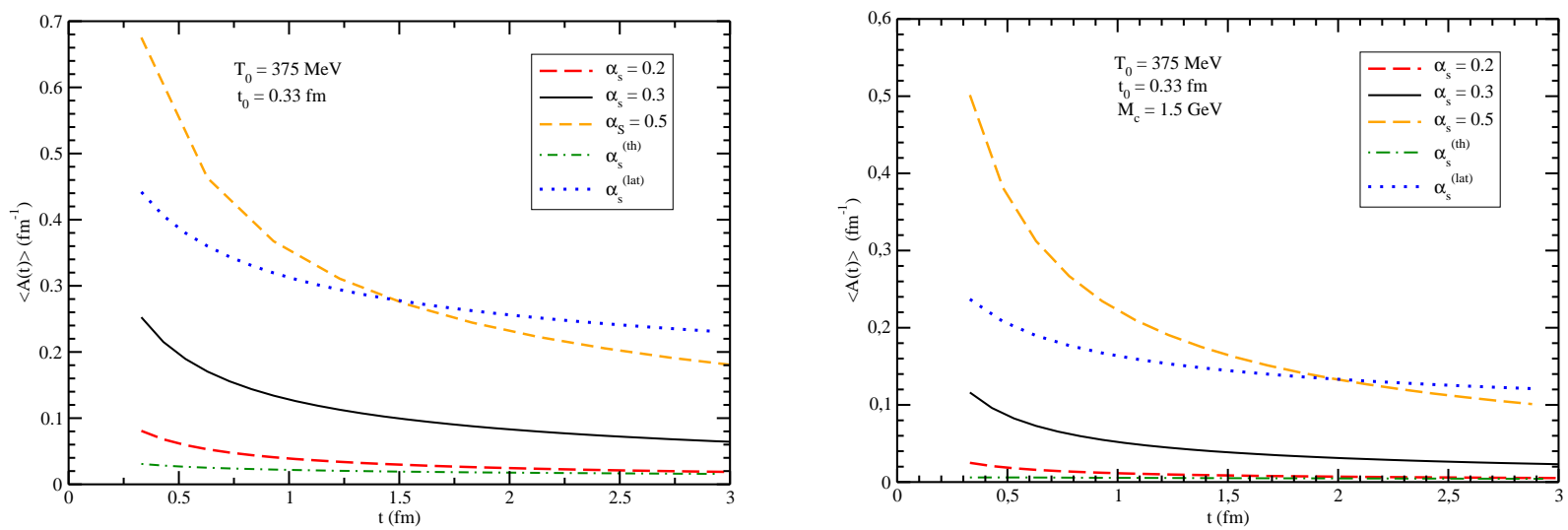

Fig. 2. Time evolution of drag coefficient for light (left panel) and heavy (charm) quarks (right panel).

coefficient $\mathcal{A}$ are show in Fig. 2 for light and heavy (charm) quarks. We present a comparison among the results for fixed $\alpha_{s}$ and running coupling from lattice and thermal QCD prescriptions. One can see that the drag coefficient is very sensitive to the modifications in the prescription used for $\alpha_{s}$. We have that when the lattice one is considered (dotted line), the coefficient becomes larger than earlier results for fixed $\alpha_{s}=0.3$ (solid line) in all the evolution of the fireball. In comparison to the $\alpha_{s}=0.5$ case (dashed line), the lattice prescription implies a smaller value of $\mathcal{A}(t)$ for small values of $t$. At large times, $\alpha_{s}^{(\text {lat })}$ becomes larger than 0.5 due to the cooling of the system, which implies a larger value of $\mathcal{A}(t)$. On the other hand, when the thermal QCD prescription is considered (dot-dashed line), the drag coefficient is smaller than the previous result, being closer to the prediction obtained assuming $\alpha_{s}=0.2$ (long-dashed line).

In Fig 3 we present the results for the fractional energy loss, as a function of the distance traveled by the partons (i.e, the time evolution of the plasma) for light and heavy quarks. Following the results for the drag coefficient, shown in Fig. 2 and discussed above, we have that $\alpha_{s}=0.5$ implies the larger amount of energy loss at small values of $L$. On the other hand, the lattice $\mathrm{QCD} \alpha_{s}$ prescription implies a large energy loss for large $L$. The thermal QCD $\alpha_{s}$ prescription provides the smallest one. Moreover, as expected, the fractional energy loss for heavy quarks is smaller than for light quarks.

In Fig. 4 we present the ratio between the fractional energy loss for heavy and light quarks. While for fixed $\alpha_{s}$ the ratio is almost constant in the range considered, for running $\alpha_{s}$ with the lattice prescription, the ratio is monotonously increasing, and the value is greater than 0.5 in all range considered; on the other hand, using the thermal prescription, the ratio is strongly suppressed when compared with the 

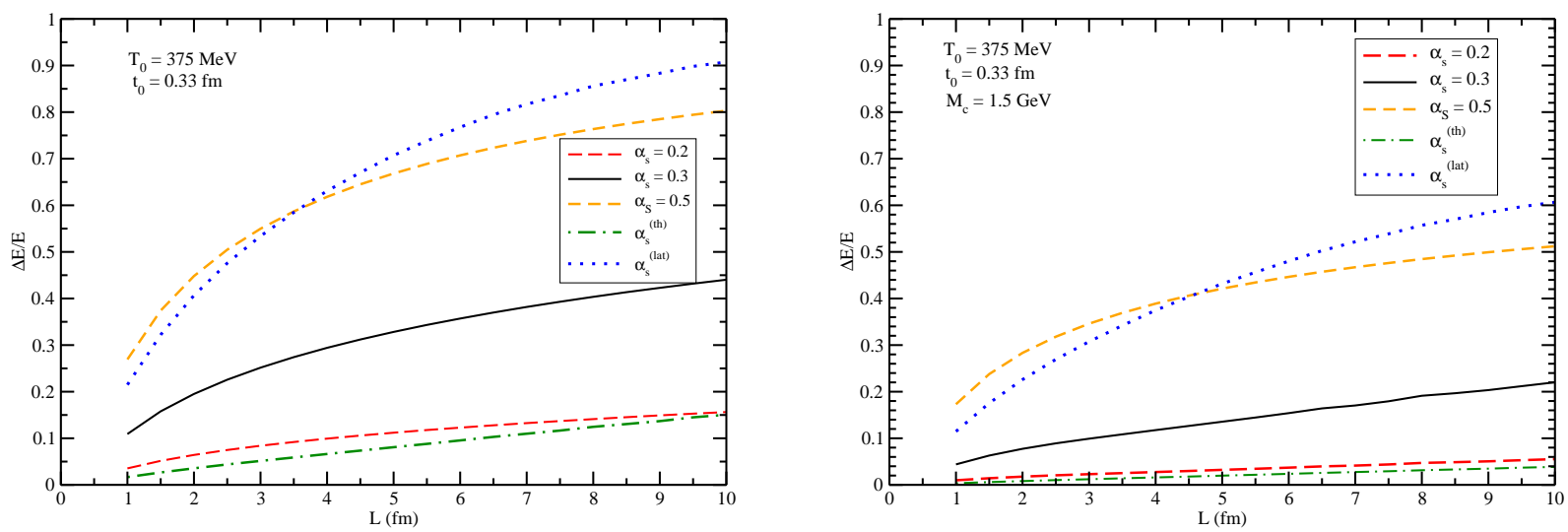

Fig. 3. Fractional energy loss as a function of the distance traveled by the light (left panel) and heavy quark (right panel).

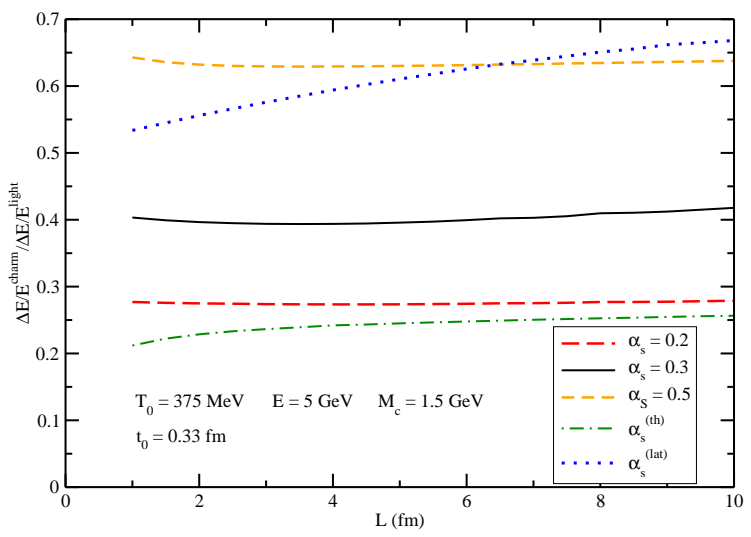

Fig. 4. Heavy-to-light ratio of the fractional energy loss.

result for fixed $\alpha_{s}$. This feature suggests that the heavy quarks lose less than $20 \%$ of the energy lost by light quarks in its path through the fireball. The predictions obtained using $\alpha_{s}=0.5$ are similar to the lattice one.

In Fig. 5 we present the results for the fractional heavy quark energy loss as a function of the incident quark energy. One have that the predictions are almost energy independent in the energy range $E \sim 5-15 \mathrm{GeV}$ for all prescriptions analyzed. This feature is due to the momentum independence of the drag coefficient, as discussed earlier 31|32. Furthermore, the magnitude of the energy loss is strongly dependent on the prescription used in the calculation.

In order to compute the $p_{\perp}$-spectra, we assume that the geometry of the heavy 
ion collision is described by a cylinder of radius $R$ and the parton moves in the transverse plane in the local rest frame. Consequently, if we consider a parton created at a point $\overrightarrow{\mathbf{r}}$ with an angle $\phi$ in the transverse direction it will travel a distance 39

$$
L(\phi)=\left(R^{2}-r^{2} \sin ^{2} \phi\right)^{1 / 2}-r \cos \phi
$$

where $\cos \phi=\hat{\overrightarrow{\mathbf{v}}} \cdot \hat{\overrightarrow{\mathbf{r}}} ; \overrightarrow{\mathbf{v}}$ is the velocity of the parton and $r=|\overrightarrow{\mathbf{r}}|$. Furthermore, we

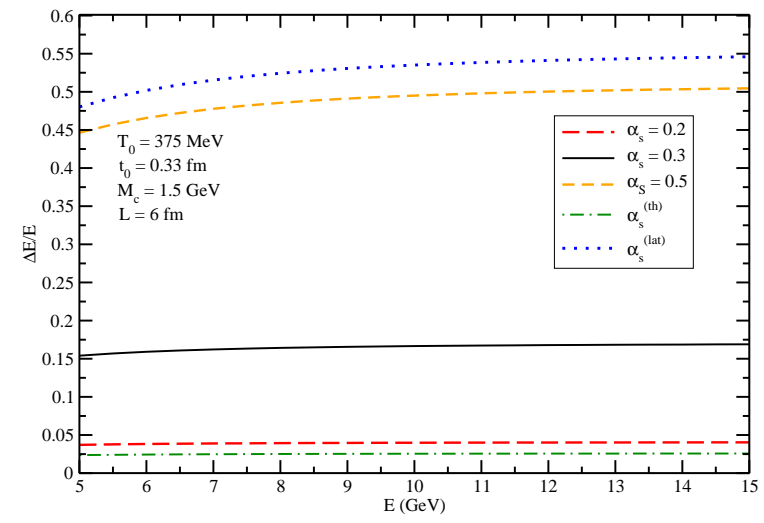

Fig. 5. Heavy quark fractional energy loss as a function of the energy of incident quark.
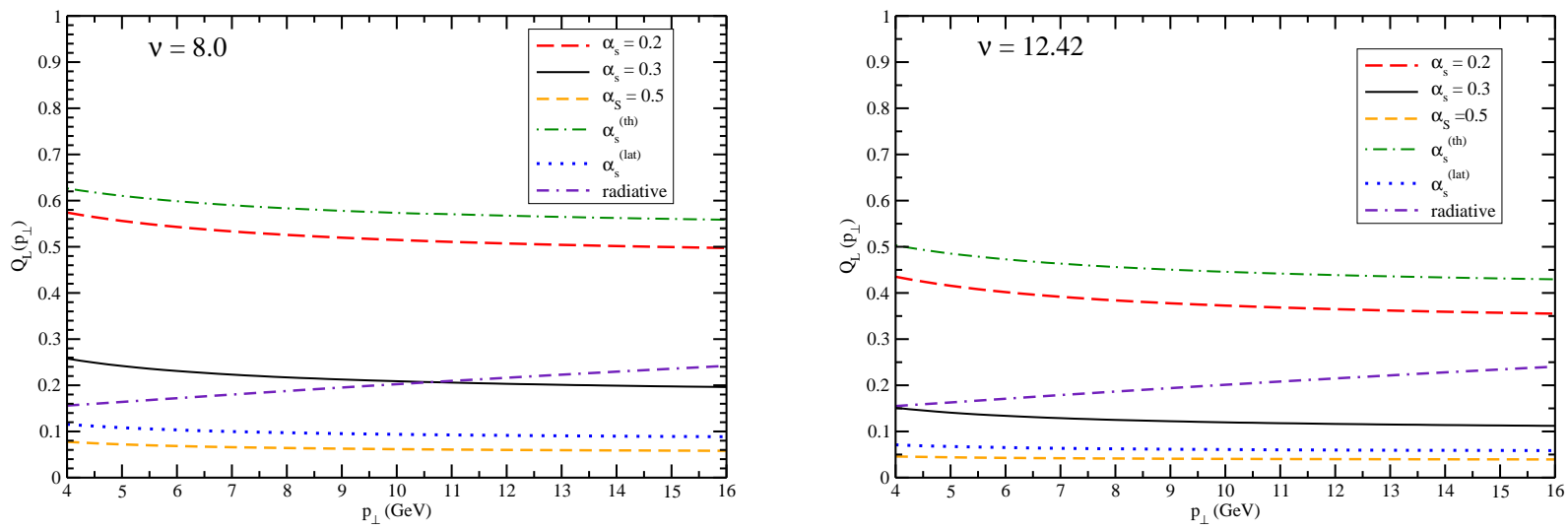

Fig. 6. Quenching factor for light quark $p_{\perp}$-spectrum. An estimative of radiative energy loss is presented for comparison. The left panel presents results with $\nu=8.0$ in the vacuum spectrum parametrization, while results with $\nu=12.42$ are presented in the right panel. 
assume the following parametrization of the $p_{\perp}$ distribution

$$
\frac{d N_{L}^{\mathrm{vac}}}{d^{2} p_{\perp}}=A\left(\frac{1}{p_{0}+p_{\perp}}\right)^{\nu}
$$

where two sets of parameters are available in the literature: $\nu=8.0$ and $p_{0}=1.75$ $\mathrm{GeV} 39$ and $\nu=12.42$ and $p_{0}=1.71 \mathrm{GeV} 48$. The results for the quenching factor for light quarks are shown in the Fig. [6 for both sets of parameters. For comparison, we present the estimate of the quenching due to the radiative energy loss, following the parametrization proposed in Ref. 39. Due to the smaller drag coefficient, the thermal QCD $\alpha_{s}$ prescription gives a high quenching factor, so the spectrum is less modified by collisional energy loss than in the case of fixed $\alpha_{s}$. At $\alpha_{s}=0.3$ and $\nu=8.0$ we have that in the high $p_{\perp}$ region, elastic and radiative energy loss are of the same order of magnitude. On the other hand, the lattice QCD $\alpha_{s}$ prescription implies a very large modification of the spectrum, similar to the predictions obtained assuming $\alpha_{s}=0.5$. Finally, if the system presents a lower value of $\alpha_{s}$ than considered in earlier calculations, the gluon bremsstrahlung becomes the dominant mechanism of energy loss again.

For heavy quarks we use the $p_{\perp}$ distribution of charmed hadrons ( $D$-mesons) produced in hadron collisions. It is experimentally found $\frac{49}{4}$ to be well described by the following simple parametrization

$$
\frac{d N_{H}^{\mathrm{vac}}}{d^{2} p_{\perp}}=C\left(\frac{1}{b M_{c}^{2}+p_{\perp}^{2}}\right)^{n / 2}
$$

where $b=1.4 \pm 0.3, n=10.0 \pm 1.2$ and $M_{c}=1.5 \mathrm{GeV}$. The quenching factor for heavy quarks is shown in Fig. 7 . The results are similar to those obtained for light quarks in Fig. 6. While the thermal QCD $\alpha_{s}$ prescription gives the higher quenching factor, the $\alpha_{s}=0.5$ one gives the smaller factor. The lattice QCD prescription implies a small quenching factor $Q_{H} \approx 0.1$, shown a strong suppression in the charm spectra when this prescription is used. Again, at smaller values of $\alpha_{s}$, higher the quenching factor due to elastic scattering in the QGP.

Recently, the ratio between the spectra of hadrons with heavy quarks and with light quarks has been proposed as a tool to investigate the medium formed in heavy ion collisions 48 . Because of its large mass, radiative energy loss for heavy quarks would be lower than for light quarks. It occurs due to combined mass effects $48 \mid 12$ : the reduction of the formation time of gluon radiation and the suppression of gluon radiation at angles smaller than the ratio of the quark mass to its energy by destructive quantum interference 50 - the dead-cone effect. The predicted consequence of these distinct radiative energy losses is an enhancement of the heavy to light spectra ratio at moderately large transverse momentum, relative to that observed in the absence of energy loss (A recent analysis for LHC energies is given in Ref. 51). As the behavior of this ratio considering collisional energy loss is still an open question, in Fig. 8 we present the ratio between heavy and light quark quenching factors, which reflects the heavy to light hadrons $(D / \pi)$ ratio, considering only collisional energy 
loss. While for values of fixed $\alpha_{s}$ smaller than 0.5 , the results show an enhancement factor close to 1.4, this enhancement is suppressed when running coupling prescriptions are considered. This feature could suggest that with an expanding cooling medium, the collisional energy loss for heavy and light quarks would be of similar magnitude. Finally, if a large value of $\alpha_{s}$ is considered the ratio can be smaller than one.

\section{Conclusions}

Before we summarize, let us discuss the assumptions made in this work in order to simplify the calculations, which may affect our results. First, we have disregarded the momentum dependence of the drag and diffusion coefficients, which contain the dynamics of the elastic collisions, replacing it by its average value. Second, the entire discussion is based on the one dimensional Fokker-Planck equation and the Bjorken model for a nuclear collision, which may not be a very realistic description, providing only a qualitative estimate. An extension for three dimensional analysis is still in discussion and may lead to a revision of our conclusions. Moreover, the inclusion of diagrams for other channels in elastic processes implies an additional uncertainty. The perturbative expressions for the (radiative and collisional) partonic energy loss, which are only known to leading order in the strong coupling constant, may get substantial corrections of higher order. A calculation of next-to-leading order corrections to energy loss in perturbative QCD would be desirable to test the stability of our results and conclusions. Finally, in order to compare our predictions with the experimental data the fragmentation of partons into hadrons should be considered and included in our calculations.

As a summary, in this paper we have investigated the dependence of the parton collisional energy loss in a QGP on the value of the strong coupling. Since the plasma

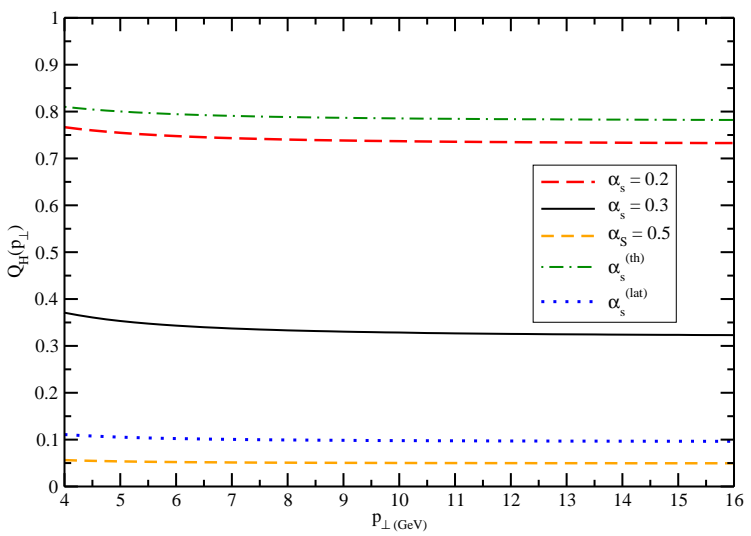

Fig. 7. Quenching factor for heavy quark $p_{\perp}$-spectrum. 


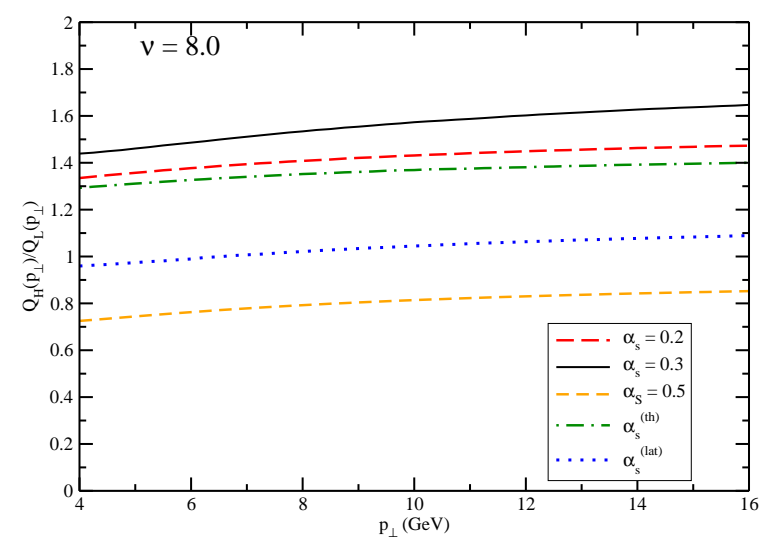

Fig. 8. Heavy-to-light quenching factor ratio.

is not a static medium, a fixed value for $\alpha_{s}$ is a crude approximation. More realistic estimates should take into account the evolution of the fireball. We have considered running coupling in the calculation, evolving it with the cooling of the QGP. From the Fokker-Planck equation, we derived the transport coefficients and related them with parton mean energy loss. The drag coefficient is found to be modified with the value of $\alpha_{s}$ considered, and it is strongly dependent on the running coupling prescription used in the analysis. The lattice one gives larger drag coefficient values, while the thermal QCD one gives smaller value, always compared with the fixed value used in previous calculations. A similar feature is verified in the mean energy loss results and quenching factors. For light quarks, we found that the radiative and collisional energy loss are of the same order of magnitude, in the high $p_{\perp}$ region, if the $\alpha_{s}$ value is larger than 0.3. For smaller values of coupling, the gluon bremsstrahlung becomes the dominant process for energy loss, again. The ratio between heavy and light quenching factors has been studied, and we have found the absence of enhancement if running coupling is used. It was a striking result, since it suggest that heavy and light quarks have the same order of magnitude for the collisional energy loss. Our results motivate a similar study in radiative parton energy loss.

\section{Acknowledgments}

This work was partially financed by CNPq and FAPERGS, Brazil.

\section{References}

1. J. Adams et al. [STAR Collaboration], Nucl. Phys. A757, 102 (2005).

2. J. Letessier and J. Rafelski, Hadrons and Quark Gluon Plasma, Cambridge Monogr. Part. Phys. Nucl. Phys. Cosmol. 18, 1 (2002). 
3. C. Y. Wong, Introduction to High-Energy Heavy Ion Collisions, Singapore, Singapore: World Scientific (1994).

4. P. Jacobs and X. N. Wang, Prog. Part. Nucl. Phys.54, 443 (2005).

5. R. Baier, D. Schiff and B. G. Zakharov, Ann. Rev. Nucl. Part. Sci.50, 37 (2000).

6. M. Gyulassy, I. Vitev, X. N. Wang and B. W. Zhang, arXiv:nucl-th/0302077.

7. A. Kovner and U. A. Wiedemann, arXiv:hep-ph/0304151

8. A. Accardi et al., arXiv:hep-ph/0310274

9. X. N. Wang, Phys. Rev. C70, 031901 (2004).

10. A. Adil and M. Gyulassy, Phys. Lett. B602, 52 (2004).

11. N. Armesto, A. Dainese, C. A. Salgado and U. A. Wiedemann, Phys. Rev. D71, 054027 (2005).

12. B. W. Zhang, E. Wang and X. N. Wang, Phys. Rev. Lett.93, 072301 (2004).

13. M. Djordjevic and M. Gyulassy, Nucl. Phys. A733, 265 (2004).

14. Q. Wang and X. N. Wang, Phys. Rev. C71, 014903 (2005).

15. H. van Hees and R. Rapp, Phys. Rev. C71, 034907 (2005)

16. G. D. Moore and D. Teaney, Phys. Rev. C71, 064904 (2005).

17. M. G. Mustafa and M. H. Thoma, Acta Phys. Hung. A22, 93 (2005) arXiv:hep-ph/0311168.

18. M. G. Mustafa, Phys. Rev. C72, 014905 (2005).

19. A. K. Dutt-Mazumder, J. e. Alam, P. Roy and B. Sinha, Phys. Rev. D71, 094016 (2005).

20. I. Vitev and M. Gyulassy, Phys. Rev. Lett.89, 252301 (2002)

21. C. A. Salgado and U. A. Wiedemann, Phys. Rev. Lett.89, 092303 (2002)

22. S. M. H. Wong, Phys. Rev. C56, 1075 (1997).

23. G. C. Nayak, A. Dumitru, L. D. McLerran and W. Greiner, Nucl. Phys. A687, 457 (2001)

24. J. D. Bjorken, Phys. Rev. D27, 140 (1983).

25. J. F. Gunion, R. Vogt, Nucl. Phys. B492, 301 (1997) .

26. P. Levai and R. Vogt, Phys. Rev. C56, 2707 (1997)

27. O. Kaczmarek, F. Karsch, F. Zantow, P. Petreczky, Phys. Rev. D70, 074505 (2004) .

28. J. Letessier, A. Tounsi and J. Rafelski, Phys. Lett. B389, 586 (1996).

29. S. M. H. Wong, Phys. Rev. C58, 2358 (1998).

30. D. d'Enterria, D. Peressounko, Eur. Phys. J. C46, 451 (2006).

31. B. Svetitsky, Phys. Rev. D37, 2484 (1988) .

32. M. G. Mustafa, D. Pal and D. K. Srivastava, Phys. Rev. C57, 889 (1998).

33. B. G. Zakharov, JETP Lett.80, 67 (2004) [Pisma Zh. Eksp. Teor. Fiz.80, 75 (2004)]

34. J. D. Bjorken, FERMILAB-PUB-82-059-THY.

35. M. H. Thoma, Phys. Lett. B273, 128 (1991).

36. E. Braaten and M. H. Thoma, Phys. Rev. D44, 2625 (1991).

37. A. Peshier, arXiv:hep-ph/0605294; arXiv:hep-ph/0607275, arXiv:hep-ph/0607299

38. R. Baier, Y. L. Dokshitzer, A. H. Mueller, and D. Schiff, JHEP0109, 033 (2001).

39. B. Müller, Phys. Rev. C67, 061901(R) (2003) .

40. S. Mrowczynski, Phys. Lett. B269, 383 (1991).

41. M. H. Thoma, Eur. Phys. J. C16, 513 (2000)

42. E. Braaten and R. D. Pisarski, Phys. Rev. Lett.64, 1338 (1990); Nucl. Phys. B337, 569 (1990); B339, 310 (1990).

43. D. B. Walton and J. Rafelski, Phys. Rev. Lett.84, 31 (2000)

44. J. Alam, B. Sinha and S. Raha, Phys. Rev. Lett.73, 1895 (1994).

45. P. Roy, J. e. Alam, S. Sarkar, B. Sinha and S. Raha, Nucl. Phys. A624, 687 (1997)

46. R. Balescu, Equilibrium and Non-Equilibrium Statistical Mechanics (Wiley, New York, 
February 2, $2008 \quad 3: 16 \quad$ WSPC/INSTRUCTION FILE mackedanz coll

16 M. B. Gay Ducati, V. P. Gonçalves and L. F. Mackedanz

1975).

47. W. E. Williams, Partial Differential Equations (Clarendon Press, Oxford, 1980).

48. Y. L. Dokshitzer and D. E. Kharzeev, Phys. Lett. B519, 199 (2001).

49. C. A. Alves et al. [E796 Collaboration], Phys. Rev. Lett.77, 2392 (1996).

50. Yu. L. Dokshitzer, V. A. Khoze, S. I. Troyan, J. Phys. G17, 1602 (1991).

51. A. Dainese, Eur. Phys. J. C33, 495 (2004). 